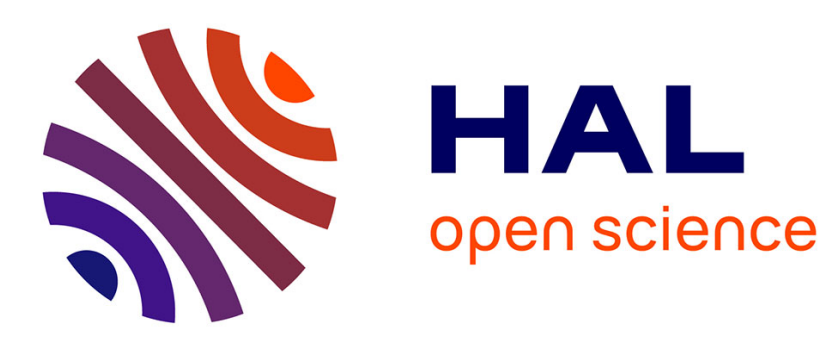

\title{
Partial and Local Knowledge for Global Efficiency of Urban Vehicular Traffic
}

Marie-Ange Lebre, Frédéric Le Mouël, Eric Ménard

\section{To cite this version:}

Marie-Ange Lebre, Frédéric Le Mouël, Eric Ménard. Partial and Local Knowledge for Global Efficiency of Urban Vehicular Traffic. IEEE 82nd Vehicular Technology Conference (VTC'2015-Fall), Sep 2015, Boston, United States. hal-01180507

\section{HAL Id: hal-01180507 https://hal.inria.fr/hal-01180507}

Submitted on 27 Jul 2015

HAL is a multi-disciplinary open access archive for the deposit and dissemination of scientific research documents, whether they are published or not. The documents may come from teaching and research institutions in France or abroad, or from public or private research centers.
L'archive ouverte pluridisciplinaire HAL, est destinée au dépôt et à la diffusion de documents scientifiques de niveau recherche, publiés ou non, émanant des établissements d'enseignement et de recherche français ou étrangers, des laboratoires publics ou privés. 


\title{
Partial and Local Knowledge for Global Efficiency of Urban Vehicular Traffic
}

\author{
Marie-Ange Lebre*t, Frédéric Le Mouël ${ }^{*}$, Eric Ménard ${ }^{\dagger}$ \\ *Université de Lyon, INSA-Lyon, CITI-INRIA, F-69621 Villeurbanne, France \\ email: $\{$ marie-ange.lebre, frederic.le-mouel $\} @$ insa-lyon.fr \\ $\dagger$ VALEO, Advanced Technology Developement, F-94000 Creteil, France \\ email: \{marie-ange.lebre, eric.menard\}@ valeo.com
}

\begin{abstract}
Intelligent transportation systems that distribute information between roadside infrastructures and vehicles are one of the most promising solutions to the problem of traffic congestion. When most existing ITS solutions are centralized and information-complete, we propose PDLAIS - a Partial, Decentralized and Locally Autonomous Strategy, tested with an application called Smooth Way, allowing drivers to customize and improve their travel time and/or fuel consumption when traveling. Our study shows that, with only $2 \%$ of independently equipped intersections, a global improvement in the fuel consumption induces a reduction of $10 \%$ of the total travel time and $25 \%$ of the global waiting time. Local decisions with pertinent partial knowledge of the network are still $5-7 \%$ close to the performance of a centralized solution.
\end{abstract}

Keywords-Intelligent Transportation Systems, Vehicular Networks, V2I, Local, Partial and Global Optimization.

\section{INTRODUCTION}

With the continuous growth of economic activities, the demand for individual mobility will increase considerably in the future. Traffic congestion is one of the leading causes of lost productivity and declining living standards in urban areas. A widely studied solution is to equip cars with onboard units and the infrastructure with roadside units that allow communication between cars (vehicle to vehicle - V2V), and with infrastructures (vehicle to infrastructure - V2I). ITS centralized solutions are largely studied and used to control traffic, but they require costly investment in installations deployed in urban areas. Decentralized solutions consider vehicles as autonomous agents but they raise the issues of safety and responsibility in the case of danger. In addition, scaling is complex.

In this context, we propose two hybrid solutions, a cross between centralized and decentralized approaches. In the first model, strategic equipped infrastructures gather only local traffic information to allow cars to take their decisions. In the second one, strategic equipped infrastructures are linked via a traffic control system and deliver partial knowledge on the whole network. The decentralized part consists of local decisions taken on the basis of information on traffic around the car. We hypothesize that our local and partial solutions will tend towards a solution with global knowledge. To validate this assumption, we propose three applications for finding the best path for the vehicle according to the user's desire to reduce his travel time - Quick Way, his consumption - Green Way, or both - Smooth Way.

The paper is organized as follows: section II gives an overview of the centralized and decentralized solutions for fuel consumption and travel time in the urban environment. Section III describes the general formulation of our hybrid models and presents the three applications available to users.
The last section describes the simulation environment and presents the results on global travel time and fuel consumption according to the users choices of application. Finally, we present our conclusion and provide the outlook for future work.

\section{RELATED WORKS}

Different kinds of approach exist for solving the problem of traffic congestion and fuel energy efficiency for vehicles. The usual choice is to implement and customize traditional algorithms such as Dijkstra, A* and Floyd. Two approaches are then used to collect information in this context: centralized and decentralized. Centralized solutions have been widely studied, for example, [1] proposed a Dijkstra-like algorithm allocating a utility function to each road used and [2] introduced energy heuristics in the $A^{*}$ algorithm. Decentralized approaches allow the exchange of traffic information between vehicles to improve travel time, fuel consumption and/or fuel emissions during a trip [3]. Biological methods have also been studied, and the ant colony algorithm has been widely used for finding optimal solutions [4]. Highly adaptable and perfect for graph-based applications, it is however susceptible to blockages and long run times.

In most cases, whether decentralized or centralized, to be optimal, these algorithms collect a great deal of information on the whole network to complete a complex large vehicular network. In [5], the authors use partial knowledge of the network learned by users in a centralized context - a server receives voice tweets from drivers on traffic events. In [6], they quantify the impact of partially-available information on the computation of route optimization.

\section{Modelling THE URBAN CONTEXT AND THE SMOOTH WAY APPLICATION}

\section{A. Urban zone modelling}

A road network can be represented by a graph $G(I, E)$ where $\left\{I_{1} \ldots I_{N}\right\}$ is a finite set of intersections and $E=$ $\left\{l_{1}, \ldots l_{m}\right\}$ is a finite set of road sections. A road section is determined by two intersections $l_{j k}=\left(I_{j}, I_{k}\right)$ and $G$ is directed, $\left(I_{j}, I_{k}\right) \neq\left(I_{k}, I_{j}\right)$. A route of a car $c$ is a directed path of road sections $R^{c}=\left\{l_{i j}, \ldots, l_{m n}\right\}$ where the $i$ th ends where the $i+1$ begins. Based on the above notations, we can define the problem formally.

The problem is to find the best path for all the vehicles in order to improve the global level of fluidity and consumption according to the driver's choice of applications. Indeed, drivers have three choices along their path, noted $P=\{T, C, T C\}$ : they can decide to improve their travel time $(P=T)$, their fuel consumption $(P=C)$ or both 
$(P=T C)$. The ideal solution of this problem requires complete knowledge of the entire network and requires heavy urban equipment [7], traffic data and computing [8]. We propose to optimize the global fluidity and consumption in the urban area with the following constraints:

- No major deterioration of the individual choice parameter $P$

- Local and/or partial knowledge of traffic in the network regarding $P$.

DEFINITION 1. Cost function.

$G$ is a weighted graph, we define it according to $P$ :

$$
\begin{array}{ccc}
\mathbf{N} & \rightarrow & {[0, \infty[} \\
t & \mapsto w_{l_{i j}}^{P}(t)
\end{array}
$$

$w_{l_{i j}}^{P}(t)$ is the mean value of $P$ on $l_{i j}$ at time $t$.

\section{DEFINITION 2. Rate of occupancy}

Let $N$ the number of vehicles on a road section $l_{i j}$ of length $L_{l_{i j}}$. The rate of occupancy (RO) is the number of vehicles per unit of length, in percentage:

$$
R O=\frac{4 . N}{L_{l_{i j}}} .100
$$

This formula of the rate of occupancy implies a relatively uniform vehicle length ( 4 meters $)^{1}$, provided for nearly filled urban areas in which traffic consists mainly of light vehicles and heavy weights of several tons. The rate of occupancy is the easiest information to gather, as it is the number of vehicles on the lane. This allows us to know when there is a traffic jam on the road section more accurately than with the speed only (for instance, one vehicle travelling at low speed can lead to a mistake in the evaluation of the traffic jam). Then, if $R O$ exceeds a threshold (see section IV), the road section is subject to a bottleneck (see section $\mathrm{V}$ ).

\section{B. Smooth Way application}

We propose an application where the driver can choose to improve his fuel consumption, travel time, or make a compromise between both.

$$
w_{l}^{T C}(t)=r w_{l}^{T}(t)+(1-r) w_{l}^{C}(t)
$$

with $0 \leq r \leq 1$ being user's choice, $P=T$ for travel time, $P=C$ for consumption and $P=T C$ for both. If $r=0$, we take the Quick Way and if $r=1$, we take the Green Way application. Smooth Way allows a compromise between the choice to arrive quickly at the destination without significant consumption during the trip.

\section{1) Quick Way:}

a) if $r=1: P$ is equal to the travel time $T$. We note $w_{l}^{T}(t)$ the average time to cross a road section $l$ at time $t$.

$$
w_{l}^{T}(t)=R O(t)+\beta(\text { Blocked })
$$

with $R O$ the current estimated rate of occupancy at time $t$ defined in section III-A.

$$
\beta(\text { Blocked })=\begin{aligned}
& \infty \text { if there is problem } \\
& 0 \text { else }
\end{aligned}
$$

\footnotetext{
${ }^{1}$ The 4-meters length hypothesis is derived from the mean value analyzed in trace. A shorter, more precise value would better the results, while our hypothesis establish upper bound results.
}

This function allows cars on the outgoing edge to send a message indicating that a lane is totally blocked because of roadworks or stopped vehicle, etc.

\section{2) Green Way:}

a) If $r=0: P$ is equal to the fuel consumption. We define the cost as:

$$
w_{l}^{C}(t)=C_{\text {average }_{l}}(t)
$$

$C_{\text {average }}(t)$ is the mean consumption on $l$ according to the number of vehicles on $l$ noted $\left.\left\|Z_{l}(t)\right\|\right)$.

$$
\begin{array}{lll}
C_{\text {average }_{l}}(t) & =\frac{\sum_{c_{i} \in Z_{l}(t)} C_{c_{i}}(t)}{\left\|Z_{l}(t)\right\|} & \text { if }
\end{array} \quad\left\|Z_{l}(t)\right\| \neq 0
$$

with $C_{c_{i}}(t)$ being the fuel consumption at time $t$ for vehicle $c_{i}$, and $C_{m i n_{l}}$ the fuel consumption for a vehicle travelling alone on $l$ at time $t$ at the maximum speed allowed on it. Finally, for the Smooth Way application, we define the total compromise between fuel consumption and travel time for a vehicle $c$ along its path $\left\{l_{1}, \ldots, l_{k_{c}}\right\}$ by:

$$
\begin{aligned}
T C_{c} & =\sum_{i=1}^{k_{c}} w_{l}^{T C}\left(t_{i}\right) \\
& =r \sum_{i=1}^{k_{c}} w_{l}^{T}\left(t_{i}\right)+(1-r) \sum_{i=1}^{k_{c}} w_{l}^{C}\left(t_{i}\right)
\end{aligned}
$$

We note time $t_{i}$ when car $c$ arrives near the intersection $I_{i}$ on its path $R^{c}=\left\{l_{1}, \ldots, l_{k_{c}}\right\}$.

\section{Partial, Decentralized Information Models}

We propose two hybrid models for information dissemination - halfway between a decentralized and a centralized approach - to study the correlation between information dissemination and traffic optimization decisions. In our model, some intersections $I^{\prime} \subseteq I$ are equipped with a communicating device. They are able to gather and disseminate information on their environment. In the first model, called PDLAIS - Partial, Decentralized and Locally Autonomous Information Strategy -, equipped intersections do not communicate their information to a central center; they are independent. Only their local traffic knowledge is exchanged within vehicles in an area limited by their range of communication. In the second model, called PCCIS Partial, Centralized and Coordinated Information Strategy -, equipped intersections communicate their information to a traffic control center, and, therefore, share partial knowledge of the whole network with the vehicles. To evaluate the potential of our models, we compare them with GCIS Global and Centralized Information Strategy - a centralized solution where each vehicle knows the state of the traffic in the network at each time step.

In our hybrid models, intersections equipped with a communicating device ensure single hop communication. Let $J \in I^{\prime}$ be an equipped intersection, and $l_{\text {out }}(J)$ its outgoing edges. By relying on V2I communication at each time step, the intersection knows the number of vehicles on its outgoing lanes. Indeed, each vehicle regularly sends a message with its identification $i d$, its position laneId and its consumption. Then, the intersection can compute the rate of occupancy $R O$ (see section III-A), the average consumption in milliliters and therefore the cost function for each parameter. The intersection considers that a high cost in time or consumption is reached at $J$ at time step $t$ if the cost function exceeds a threshold $\xi_{l}^{P}$ :

$$
\exists l \in l_{\text {out }}(J), w_{l}^{P}(t) \geq \xi_{l}^{P}
$$


with $\xi_{l}^{T}$ being a fixed threshold with a rate of occupancy according to the shape of the road while $\xi_{l}^{C}$ is computed with the consumption function $I V$.

$$
\begin{aligned}
& \xi_{l}^{C}=1.5 * C_{m_{i n}} \\
& \xi_{l}^{T}=\text { Limit of Rate of Occupancy }
\end{aligned}
$$

The value $C_{\text {min }_{l}}$ corresponds to the fuel consumption for a vehicle travelling alone on $l$ at time $t$ at maximum speed. We consider that vehicles have a consumption around 10 liters for 100 kilometers at $150 \mathrm{~km}$ per hour, and, with this hypothesis, we have the following equation for the consumption $C$ :

$$
C=\frac{2 L_{l}}{V}+\frac{13 V^{2}}{33750}
$$

$L_{l}$ is the length of $l$. If $V=V_{\max }$ we have an estimation of the fuel consumption when the car is alone on the edge ${ }^{2}$. At each time step, the equipped intersection sends the cost function $w_{l}^{P}$ for each outgoing edge $l \in l_{\text {out }}(J)$. In PDLAIS, the intersection sends this value to the approaching vehicles; in PCCIS, it sends this information to the traffic control center. When the cost function exceeds the threshold in time or consumption on $l$, the intersection sends $\infty$ in spite of the real cost function $w_{l}^{P}(t)$. These thresholds permit the intersection to control the traffic, and, using the information, the vehicles recompute their itinerary with a Dijkstra algorithm.

At the beginning of its travel, a car $c$ computes its route $R^{c}$ offline with Dijkstra's algorithm (the weights on a road correspond to the travel time with the maximum speed allowed on it). In PDLAIS, when the vehicle crosses an equipped intersection, it recomputes its shortest path according to the user's choice of application and the traffic information sent by the intersection. Thus, the number of recomputed itineraries is bound by the number of equipped junctions that a vehicle crosses during its travel. In PCCIS, the traffic control center sends this local information to all the vehicles. Therefore, all the vehicles recompute on the basis of the partial information on the whole network received by the traffic control center. To sum up, the vehicles have local and partial knowledge with the first method and partial knowledge of the whole network with the second. Therefore, the quantity of information sent in the two hybrid models is different and lower than the centralized solutionto avoid data saturation in the network. In this context, the number of equipped intersections in the network plays an important role in our models. Logically, intersections with daily congestion problems should be chosen. Indeed, if there is no congestion at an intersection, the traffic is fluid and $w_{l}^{P}(t)$ will never be equal to $\infty$; consequently, the vehicle will probably never change its route. We want to find the smallest number of intersections that tend to the centralized solution in terms of total travel time and total fuel consumption.

\section{Simulation And Results}

\section{A. Environment and hypothesis}

We use a simulator of urban mobility: SUMO version 0.22 - an open source microscopic and continuous road traffic simulation package, coupled with a Traffic Control

\footnotetext{
${ }^{2}$ All vehicles have the same fuel consumption model. In a future study, we will consider each vehicle having its own consumption function.
}

Interface (TraCI) to control the behavior of vehicles during simulation runtime. For a realistic scenario, we used the TAPASCologne dataset [9] - reproducing the urban traffic in the city of Cologne as realistically as possible - with vehicles equipped with wireless 802.11 p connection.

\begin{tabular}{|l|l|}
\hline Dimension of the map & $1400 \mathrm{~m} \times 666 \mathrm{~m}$ \\
\hline Time & 7 am to $7: 15 \mathrm{am}$. \\
\hline Number of vehicles & 1800 \\
\hline Number of intersections & 64 \\
\hline Speed of fast roads & 90,80 and $70 \mathrm{~km} / \mathrm{h}$ \\
\hline Beginning of congestion on fast roads & Rate of occupancy $>16 \%$ \\
\hline Speed on slow roads & 50 and $35 \mathrm{~km} / \mathrm{h}$ \\
\hline Beginning of congestion on slow roads & Rate of occupancy $>20 \%$ \\
\hline
\end{tabular}

TABLE I: Parameters of the simulations

To find the minimum number of intersections and the best parameter $r$ in the Smooth Way, we test our models in different situations. We consider four new configurations on the same map with random departures and destinations. We consider the same number of vehicles and the same distance traveled in order to be close to the real scenario. We called these five configurations: Map1Cologne (real case), Map2, Map3, Map4 and Map5.

In the city, different road shapes - slow and fast - are characterized by different levels of services in function of their occupancy rate. The limit rate of occupancy presented in the Table I represents our time threshold $\xi_{l}^{T}$ defined in section IV.

In an urban area, trips are daily, consequently some intersections are always more congested than others during a day. In our proposal, we decide to equip some intersections without traffic lights according to their level of congestion. During observation of the urban area without wireless device, we count how many times the outgoing roads have a higher cost function in time than the threshold $\xi_{l}^{T}$. We select all the intersections with a count higher than 0 - an average of 64 intersections on the 5 maps - to be equipped, since there is no point in considering intersections with a null count as no re-routing will be done at these intersections.

\section{B. Results}

In the following simulations, we compare our two hybrid models PDLAIS and PCCIS to the centralized model GCIS. We determine the number of intersections to be equipped. We compare these models considering the Smooth Way application to determine the best $r$ value to optimize travel time and/or fuel consumption. Stability of the results are tested with different driver behaviors and different wireless communication conditions.

1) Number of equipped intersections and $r$ for Smooth Way: First, we want to find out how many intersections we have to choose in our PDLAIS model in order to be efficient for travel time, fuel consumption and waiting time, by applying the recommended path Smooth Way. To achieve this, we decrease the number of equipped intersections as a function of their daily traffic problem. The results are presented in Figure 1 . The value 1 on the $\mathrm{Z}$ axis represent the reference value of route planning without any ITS recommendation system - no knowledge. For each number of equipped intersections, we test different values of $r$ for the Smooth Way, considering that all the vehicles chose the same $r$. We compute, for each map, the mean of waiting time, fuel consumption or travel duration. The red surface corresponds to the comparison between PDLAIS and the reference value without knowledge, the blue surface 
describes the comparison between GCIS and the reference value without knowledge.

Only a small number of congested intersections need to be equipped, around $2 \%$ (on average: 3 to 6 intersections out of 64 intersections), and perform results $5-7 \%$ close to the centralized solution: $10 \%$ gain in duration, $25 \%$ gain in waiting time, and even perform $1-2 \%$ close with $3 \%$ gain in fuel consumption. Another interesting result is that from $20 \%$ to $30 \%$ of equipped intersections, the introduced coordination noise produces worst results than the reference value without any knowledge.

$r=0.1$ and $r=0.5$ appears to be the best values for the three metrics considering $2 \%$ of equipped intersections. Parameters $r=0.1$ and $r=0.5$ achieve better results for fuel consumption, duration and waiting time than $r=1$, which is supposed to be the "Quick Way". They are also better than $r=0$ - the "Green Way" for duration and waiting time. They allow reaching a compromise with almost the best duration and the better fuel consumption while obtaining a saving of up to $25 \%$ for waiting time.

$r=0.1$ can even slightly outperform $r=0.5$ for fuel consumption, but the variance (see the table II) is higher than for $r=0.5$, meaning some users are penalized and lost time for the community in a reasonable manner.

\begin{tabular}{|l|cccccccccc|}
\hline$\sigma^{2}$ & \multicolumn{10}{|c|}{ Percentage of equipped intersection } \\
& $100 \%$ & $50 \%$ & $40 \%$ & $30 \%$ & $20 \%$ & $10 \%$ & $8 \%$ & $5 \%$ & $2 \%$ \\
\hline Duration & & & & & & & & & \\
r=0.1 & 0.25 & 0.21 & 0.23 & 0.13 & 0.18 & 0.17 & 0.15 & 0.18 & 0.18 \\
r=0.5 & 0.25 & 0.20 & 0.17 & 0.14 & 0.13 & 0.08 & 0.08 & 0.07 & 0.10 \\
\hline Fuel & & & & & & & & & \\
r=0.1 & 0.04 & 0.05 & 0.04 & 0.04 & 0.02 & 0.03 & 0.02 & 0.01 & 0.02 \\
r=0.5 & 0.05 & 0.04 & 0.05 & 0.03 & 0.03 & 0.03 & 0.03 & 0.01 & 0.01 \\
\hline Wait & & & & & & & & & \\
r=0.1 & 0.59 & 0.38 & 0.58 & 0.30 & 0.34 & 0.25 & 0.20 & 0.25 & 0.29 \\
r=0.5 & 0.51 & 0.47 & 0.37 & 0.26 & 0.18 & 0.11 & 0.12 & 0.15 & 0.14 \\
\hline
\end{tabular}

TABLE II: Standard deviation for duration, fuel consumption and waiting time for $\mathrm{r}=0.1$ and $\mathrm{r}=0.5$

2) PDLAIS vs PCCIS: We use $r=0.1$ with $2 \%$ equipped intersections to detail the difference in performance between the two hybrid solutions. As the Figure 2 (a) shows, PDLAIS and PCCIS are similar in terms of performance: duration and waiting time are the same with the same deviation; PCCIS is $2 \%$ slightly better rather than PDLAIS for fuel consumption, but also with a $2 \%$ standard deviation.

In terms of transmitted data, PCCIS and PDLAIS require substantially less data than GCIS - only $4.04 \%$ - cf Figure 2 (b). Then in PCCIS, all vehicles receive back data from the traffic control center in the same way as GCIS, while in PDLAIS only $6.7 \%$ of the vehicles receive back these data. Therefore, with PDLAIS, a huge saving in the amount of data can be achieved, as well as in the number of initiated communications, avoiding saturation and allowing the transmission of traffic information in real time. To conclude, we focus the last simulations only on the PDLAIS model.

3) Stability of the value $r=0.1$ on PDLAIS: We test the stability of the value $r=0.1$ by introducing different driver behaviors - i.e having different $r$ value at different time. To achieve this, we evaluate a random $r$ and a normal distribution around the 0.1 mean value. The circles on the Figure 3 gather the results according to the considered maps. We notice that performance can greatly vary between the maps and the configuration of itineraries, but only for better gains. Globally, fuel consumption is stable with a $2-3 \%$ variation, duration is stable with a $5 \%$ variation, and waiting
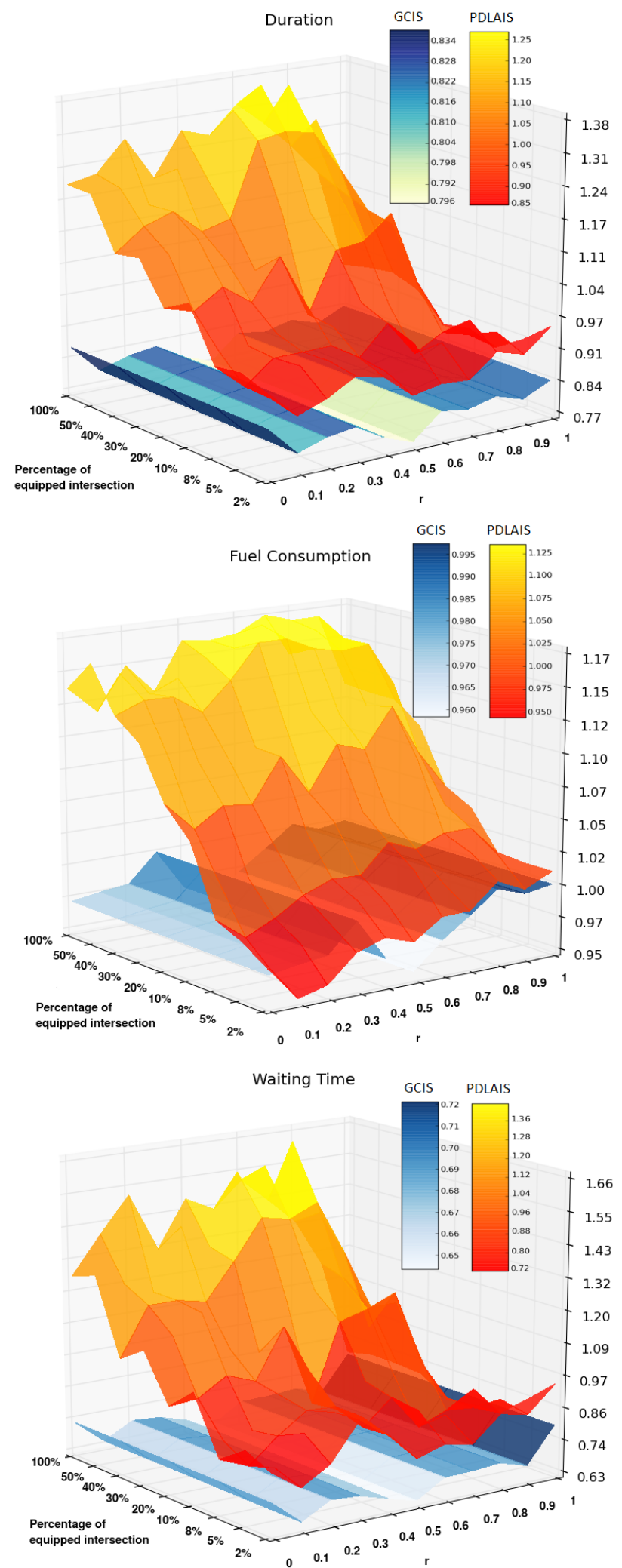

Fig. 1: Duration, fuel consumption and waiting time ratio according to $r \in[0,1]$ on Smooth Way and intersection infrastructure

time can vary a bit more with a $10 \%$ variation. The results with the normal distributions mean that $r=0.1$ is not affected by the other value of $r$, thus it is a stable value. Similar results are also obtained for the random $r$, so an equiprobable distribution is also a good solution. 


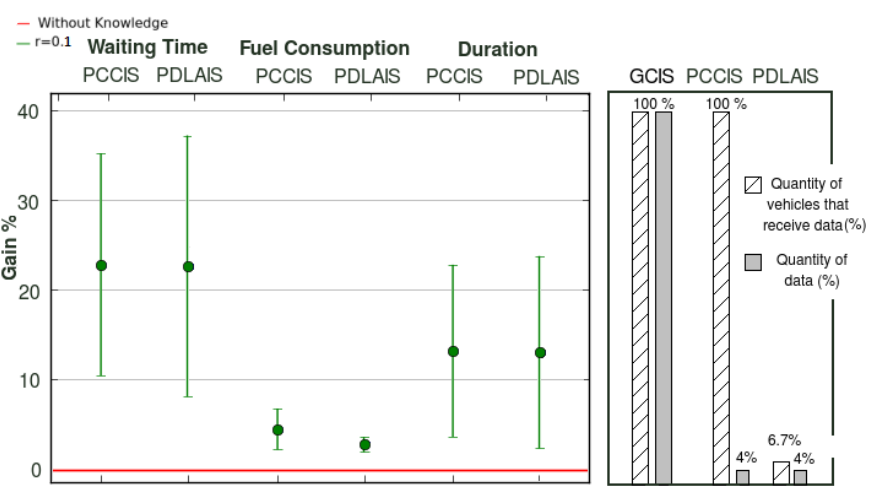

Fig. 2: Comparison between PDLAIS and PCCIS

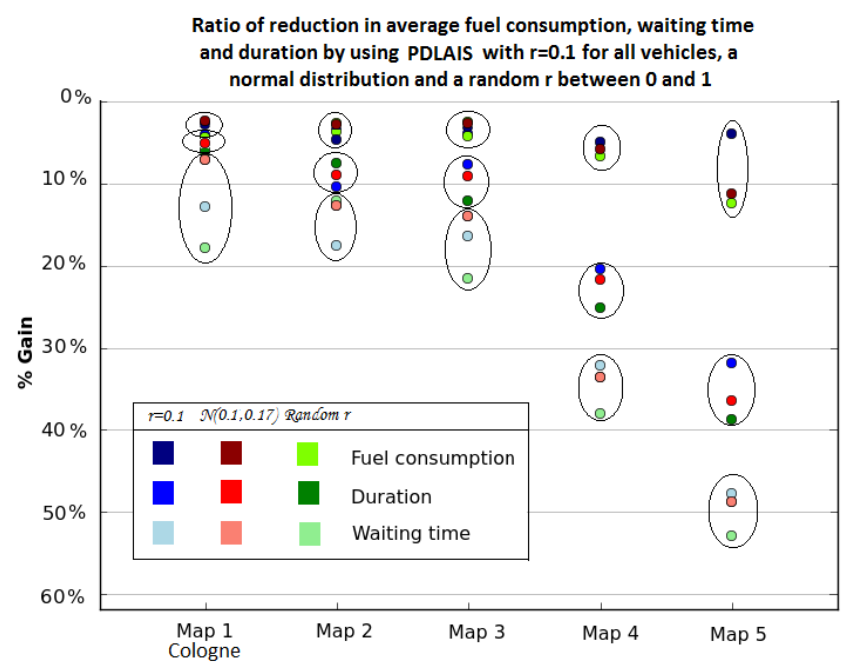

Fig. 3: Different distribution of $r$ with PDLAIS

4) Range of communication on PDLAIS: To complete our study, we evaluate the impact of the range of communication and the packet loss on the PDLAIS model. Simulations do not take into account the buildings or other obstacles for propagation, but, even if not totally realistic, they provide an idea of the impact of the communication radius. We compare the two following situations: in the first, the vehicles have a probability $P(d)$ to loose the packets thanks to the equation (12) and, in the second one, the vehicles receive the message at $d$ meters from the intersections with a probability equal to $P(d)=1$. We use the Nakagami-based formula for clear channel packet loss probability which is characterized by the radio range $D$ and the distance $d$ of the vehicle from the intersection (we consider that the intersection has a range of communication equal to $D=250$ meters):

$$
P(d)=1-\mathrm{e}^{-3 \cdot\left(\frac{d}{D}\right)^{2}} \cdot\left(1+3 \cdot\left(\frac{d}{D}\right)^{2}+4 \cdot 5 \cdot\left(\frac{d}{D}\right)^{4}\right)
$$

We still evaluate the average of each metrics on the five maps. Figure 4 shows the results. For larger distances greater than 200 meters - results are stable: the needed information is local and immediate and having the information earlier is finally not significant, and it's even better to loss packets - $P(d)$ gains are $3 \%$ better than $P(d)=1$. Then, receiving the information close to the intersection is significant: $P(d)$ gains are $3-4 \%$ worse than $P(d)=1$ for duration and waiting time only. Fuel consumption gap is negligible with short distances.

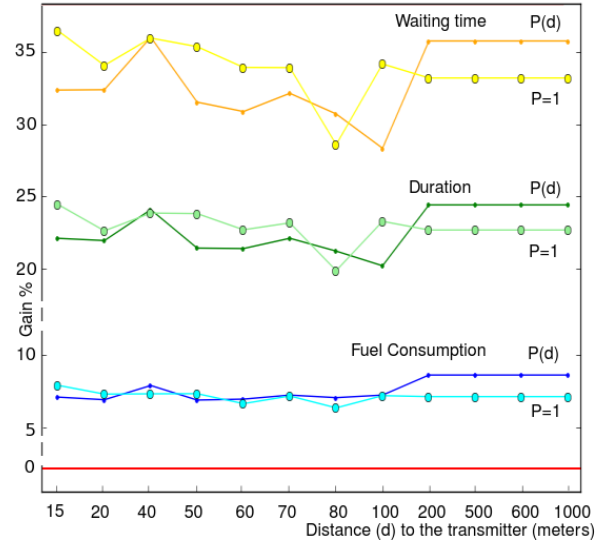

Fig. 4: Impact of the range of communication and packet loss

\section{CONCLUSION AND FUture WORK}

The results obtained in our study highlight two interesting points. First, an urban area can be connected at strategic points in the context of traffic control. With only $2 \%$ of equipped intersections, local decisions decrease the travel time, waiting time and fuel consumption over the whole area. The improvement of traffic conditions is close to the one achieved with a centralized solution with significantly fewer transmitted data. Second, by focusing on $90 \%$ fuel consumption thanks to Smooth Way $(r=0.1)$, the travel time and the waiting time are quite close to the better values. If all the drivers improve only their travel time $(r=0)$ or their fuel consumption $(r=1)$, they deteriorate the global travel time. This model could be extended to achieve more accurate results, e.g. with PDCIS - Partial Decentralized Coordinated Information System - where the strategic intersections would be able to communicate together.

\section{REFERENCES}

[1] R. Doolan and G. Muntean, "VANET-enabled eco-friendly road characteristics-aware routing for vehicular traffic," in IEEE Vehicular Technology Conference, VTC Spring, 2013, pp. 1-5.

[2] M. Sachenbacher, M. Leucker, A. Artmeier, and J. Haselmayr, "Efficient energy-optimal routing for electric vehicles," in 25th Conference on Artificial Intelligence, AAAI, 2011, pp. 1402-1407.

[3] I.-C. Chang, H.-T. Tai, F.-H. Yeh, D.-L. Hsieh, and S.-H. Chang, "A VANET-based $\mathrm{A}^{*}$ route planning algorithm for travelling time and energy efficient gps navigation," International Journal of Distributed Sensor Networks, 2013.

[4] R. Claes and T. Holvoet, "Ant colony optimization applied to route planning using link travel time predictions," in IEEE International Symposium on Parallel and Distributed Processing Workshops and PhD Forum. IEEE, 2011, pp. 358-365.

[5] W. Sha, D. Kwak, B. Nath, and L. Iftode, "Social vehicle navigation: integrating shared driving experience into vehicle navigation," in 14th Workshop on Mobile Computing Systems and Applications, HotMobile. ACM, 2013.

[6] L. Codeca, R. Frank, and T. Engel, "Traffic routing in urban environments: The impact of partial information," in 17th International Conference on Intelligent Transportation Systems, ITSC. IEEE, 2014, pp. 2511-2516.

[7] M. Jerbi, S. M. Senouci, T. Rasheed, and Y. Ghamri-Doudane, "An infrastructure-free traffic information system for vehicular networks," in IEEE Vehicular Technology Conference, VTC, 2007, pp. 2086-2090.

[8] J. Zhou, R. Q. Hu, and Y. Qian, "A scalable vehicular network architecture for traffic information sharing," Selected Areas in Communications, IEEE Journal on, vol. 31, no. 9, pp. 85-93, 2013.

[9] S. Uppoor and M. Fiore, "Large-scale urban vehicular mobility for networking research," IEEE Vehicular networking conference, VNC, pp. 62-69, 2011. 